\title{
Global Attractors and Dimension Estimation of the 2D Generalized MHD System with Extra Force
}

\author{
Zhaoqin Yuan, Liang Guo, Guoguang Lin* \\ Department of Mathematics, Yunnan University, Kunming, China \\ Email: yuanzq091@163.com, gglin@ynu.edu.cn
}

Received 16 March 2015; accepted 26 April 2015; published 29 April 2015

Copyright (C) 2015 by authors and Scientific Research Publishing Inc.

This work is licensed under the Creative Commons Attribution International License (CC BY).

http://creativecommons.org/licenses/by/4.0/

(c) (;) Open Access

\section{Abstract}

In this paper, firstly, some priori estimates are obtained for the existence and uniqueness of solutions of a two dimensional generalized magnetohydrodynamic (MHD) system. Then the existence of the global attractor is proved. Finally, the upper bound estimation of the Hausdorff and fractal dimension of attractor is got.

Keywords

MHD System, Existence, Global Attractor, Dimension Estimation

\section{Introduction}

In this paper, we study the following magnetohydrodynamic system:

$$
\left\{\begin{array}{l}
\frac{\partial u}{\partial t}+(u \cdot \nabla) u-(v \cdot \nabla) v+\gamma A^{2 \alpha} u=f(x) \\
\frac{\partial v}{\partial t}+(u \cdot \nabla) v-(v \cdot \nabla) u+\eta A^{2 \beta} v=g(x) \\
\nabla u=\nabla v=0 \\
(u, v)(x, 0)=\left(u_{0}, v_{0}\right)(x) \\
\left.u(x, t)\right|_{\partial \Omega}=\left.v(x, t)\right|_{\partial \Omega}=0
\end{array}\right.
$$

${ }^{*}$ Corresponding author. 
here $\Omega \subset R^{2}$ is bounded set, $\partial \Omega$ is the bound of $\Omega$, where $u$ is the velocity vector field, $v$ is the magnetic vector field, $\gamma, \eta>0, \alpha, \beta>\frac{n}{2}$ are the kinematic viscosity and diffusivity constants respectively. $A=(-\Delta)$. Let $\|\cdot\|=\|\cdot\|_{L^{2}(\Omega)},\|\cdot\|_{\infty}=\|\cdot\|_{L^{\infty}(\Omega)}$.

When $\alpha=\beta=1$, problem (1.1) reduces to the MHD equations. In particular, if $\gamma=\eta=0$, problem (1.1) becomes the ideal MHD equations. It is therefore reasonable to call (1.1) a system of generalized MHD equations, or simply GMHD. Moreover, it has similar scaling properties and energy estimate as the Navier-Stokes and MHD equations.

The solvability of the MHD system was investigated in the beginning of 1960s. In particular in [1]-[4] the global existence of weak solutions and local in time well-posedness was proved for various initial boundary value problems. However, similar to the situation with the Navier-Stokes equations, the problem of the global smooth solvability for the MHD equations is still open.

Analogously to the case of the Navier-Stokes system (see [5]-[8]) we introduce the concept of suitable weak solutions. We prove the existence of the global attractor (see [9]) and getting the upper bound estimation of the Hausdorff and fractal dimension of attractor for the MHD system.

\section{The Priori Estimate of Solution of Problem (1.1)}

Lemma 1. Assume $\left(u_{0}, v_{0}\right) \in L^{2}(\Omega) \times L^{2}(\Omega),(f(x), g(x)) \in L^{2}(\Omega) \times L^{2}(\Omega)$, so the smooth solution $(u(x, t), v(x, t))$ of problem (1.1) satisfies

$$
\left(\|u\|^{2}+\|v\|^{2}\right) \leq\left(\left\|u_{0}\right\|^{2}+\left\|v_{0}\right\|^{2}\right) \mathrm{e}^{-a t}+\frac{1}{a^{2}}\left(\|f\|^{2}+\|g\|^{2}\right) .
$$

Proof. We multiply $u$ with both sides of the first equation of problem (1.1) and obtain

$$
\frac{1}{2} \frac{\mathrm{d}}{\mathrm{d} t}\|u\|^{2}+(u \nabla u, u)-(v \nabla v, u)+\gamma\left\|A^{\alpha} u\right\|^{2}=(f(x), u),
$$

We multiply $v$ with both sides of the second equation of problem (1.1) and obtain

$$
\frac{1}{2} \frac{\mathrm{d}}{\mathrm{d} t}\|v\|^{2}+(u \nabla v, v)-(v \nabla u, v)+\eta\left\|A^{\beta} v\right\|^{2}=(g(x), v),
$$

According to $b(u, u, v)=-b(u, v, u)$, we obtain

$$
b(u, u, u)=b(u, v, v)=0, b(v, v, u)=-b(v, u, v),
$$

According to (2.1) $+(2.2)$, so we obtain

$$
\frac{1}{2} \frac{\mathrm{d}}{\mathrm{d} t}\left(\|u\|^{2}+\|v\|^{2}\right)+\gamma\left\|A^{\alpha} u\right\|^{2}+\eta\left\|A^{\beta} v\right\|^{2}=(f(x), u)+(g(x), v),
$$

According to Poincare and Young inequality, we obtain

$$
\begin{gathered}
\left\|A^{\alpha} u\right\|^{2} \geq \lambda_{1}^{2 \alpha}\|u\|^{2},\left\|A^{\beta} v\right\|^{2} \geq \lambda_{1}^{2 \beta}\|v\|^{2}, \\
|(f(x), u)| \leq\|u\|\|f\| \leq \frac{\gamma \lambda_{1}^{2 \alpha}}{4}\|u\|^{2}+\frac{1}{\gamma \lambda_{1}^{2 \alpha}}\|f\|^{2}, \\
|(g(x), v)| \leq\|v\|\|g\| \leq \frac{\eta \lambda_{1}^{2 \beta}}{4}\|v\|^{2}+\frac{1}{\eta \lambda_{1}^{2 \beta}}\|g\|^{2},
\end{gathered}
$$

From (2.5)-(2.7), we obtain

$$
\begin{aligned}
& \frac{1}{2} \frac{\mathrm{d}}{\mathrm{d} t}\left(\|u\|^{2}+\|v\|^{2}\right)+\frac{\gamma}{2}\left\|A^{\alpha} u\right\|^{2}+\frac{\gamma \lambda_{1}^{2 \alpha}}{2}\|u\|^{2}+\frac{\eta}{2}\left\|A^{\beta} v\right\|^{2}+\frac{\eta \lambda_{1}^{2 \beta}}{2}\|v\|^{2} \\
& \leq \frac{\gamma \lambda_{1}^{2 \alpha}}{4}\|u\|^{2}+\frac{1}{\gamma \lambda_{1}^{2 \alpha}}\|f\|^{2}+\frac{\eta \lambda_{1}^{2 \beta}}{4}\|v\|^{2}+\frac{1}{\eta \lambda_{1}^{2 \beta}}\|g\|^{2},
\end{aligned}
$$




$$
\frac{\mathrm{d}}{\mathrm{d} t}\left(\|u\|^{2}+\|v\|^{2}\right)+\frac{\gamma \lambda_{1}^{2 \alpha}}{2}\|u\|^{2}+\frac{\eta \lambda_{1}^{2 \beta}}{2}\|v\|^{2} \leq \frac{2}{\gamma \lambda_{1}^{2 \alpha}}\|f\|^{2}+\frac{2}{\eta \lambda_{2}^{2 \beta}}\|g\|^{2},
$$

Let $a=\min \left\{\frac{\gamma \lambda_{1}^{2 \alpha}}{2}, \frac{\eta \lambda_{1}^{2 \beta}}{2}\right\}$, according that we obtain

$$
\frac{\mathrm{d}}{\mathrm{d} t}\left(\|u\|^{2}+\|v\|^{2}\right)+a\left(\|u\|^{2}+\|v\|^{2}\right) \leq \frac{1}{a}\left(\|f\|^{2}+\|g\|^{2}\right) .
$$

Using the Gronwall's inequality, the Lemma 1 is proved.

Lemma 2. Under the condition of Lemma 1 , and $\left(u_{0}, v_{0}\right) \in H^{2 \alpha}(\Omega) \times H^{2 \beta}(\Omega)$,

$(f(x), g(x)) \in L^{2}(\Omega) \times L^{2}(\Omega), \quad \alpha>\frac{n}{2}, \quad \beta>\frac{n}{2}$, so the solution $\left(A^{\alpha} u, A^{\beta} v\right)$ of problem (1.1) satisfies

$$
\left(\left\|A^{\alpha} u\right\|^{2}+\left\|A^{\beta} v\right\|^{2}\right) \leq\left(\left\|A^{\alpha} u_{0}\right\|^{2}+\left\|A^{\beta} v_{0}\right\|^{2}\right) \mathrm{e}^{-a t}+\frac{1}{a^{2}}\left(\left\|A^{\alpha} f\right\|^{2}+\left\|A^{\beta} g\right\|^{2}\right)+\frac{2}{a} C_{10} .
$$

Proof. For the problem (1.1) multiply the first equation by $A^{2 \alpha} u$ with both sides, for the problem (1.1) multiply the second equation by $A^{2 \beta} v$ with both sides and obtain

$$
\begin{gathered}
\left\{\begin{array}{c}
\frac{1}{2} \frac{\mathrm{d}}{\mathrm{d} t}\left\|A^{\alpha} u\right\|^{2}+\left(u \nabla u, A^{2 \alpha} u\right)-\left(v \nabla v, A^{2 \alpha} u\right)+\gamma\left\|A^{2 \alpha} u\right\|^{2}=\left(f, A^{2 \alpha} u\right), \\
\frac{1}{2} \frac{\mathrm{d}}{\mathrm{d} t}\left\|A^{\beta} v\right\|+\left(u \nabla v, A^{2 \beta} v\right)-\left(v \nabla u, A^{2 \beta} v\right)+\eta\left\|A^{2 \beta} v\right\|^{2}=\left(g, A^{2 \beta} v\right) .
\end{array}\right. \\
\left|\left(u \nabla u, A^{2 \alpha} u\right)\right| \leq \int_{\Omega}\left|u\|\nabla u\| A^{2 \alpha} u\right| \mathrm{d} x \leq\|u\|_{L^{4}}\|\nabla u\|_{L^{4}}\left\|A^{2 \alpha} u\right\|,
\end{gathered}
$$

According to the Sobolev's interpolation inequalities,

$$
\begin{gathered}
\|u\|_{L^{4}} \leq C_{0}\|\Delta u\|^{\frac{n}{16 \alpha}}\|u\|^{1-\frac{n}{16 \alpha}},\|\nabla u\|_{L^{4}} \leq C_{1}\|\Delta u\|^{\frac{4+n}{16 \alpha}}\|u\|^{1-\frac{4+n}{16 \alpha}}, \\
\|v\|_{L^{4}} \leq C_{2}\|\Delta v\|^{\frac{n}{16 \beta}}\|v\|^{1-\frac{n}{16 \beta}},\|\nabla v\|_{L^{4}} \leq C_{3}\|\Delta v\|^{\frac{4+n}{16 \beta}}\|v\|^{1-\frac{4+n}{16 \beta}},
\end{gathered}
$$

According to (2.9)-(2.10), we have

$$
\begin{aligned}
\|u\|_{L^{4}}\|\nabla u\|_{L^{4}}\left\|A^{2 \alpha} u\right\| & \leq\|\Delta u\|^{\frac{2+n}{8 \alpha}}\|u\|^{2-\frac{2+n}{8 \alpha}}\left\|A^{2 \alpha} u\right\| \leq C_{4}\|\Delta u\|^{\frac{2+n}{8 \alpha}}\left\|A^{2 \alpha} u\right\| \\
& \leq C_{5}\left\|A^{2 \alpha} u\right\|^{1+\frac{2+n}{8 \alpha}} \leq \frac{\gamma}{16}\left\|A^{2 \alpha} u\right\|^{2}+C_{6},
\end{aligned}
$$

Here

$$
C_{6} \geq C_{5}^{\frac{8 \alpha+2+n}{8 \alpha-2-n}}
$$

In a similar way, we can obtain

$$
\begin{aligned}
\left|\left(v \nabla v, A^{2 \alpha} u\right)\right| & \leq\|v\|_{L^{4}}\|\nabla v\|_{L^{4}}\left\|A^{2 \alpha} u\right\| \leq\|\Delta v\|^{\frac{2+n}{8 \beta}}\|v\|^{2-\frac{2+n}{8 \beta}}\left\|A^{2 \alpha} u\right\| \\
& \leq C_{7}\|\Delta v\|^{\frac{2+n}{8 \beta}}\left\|A^{2 \alpha} u\right\| \leq C_{8}\left\|A^{2 \alpha} u\right\|\left\|A^{2 \beta} v\right\|^{\frac{2+n}{8 \beta}} \\
& \leq \frac{\gamma}{16}\left\|A^{2 \alpha} u\right\|^{2}+\frac{4 C_{8}^{2}}{\gamma}\left\|A^{2 \beta} v\right\|^{\frac{2+n}{4 \beta}} \\
& \leq \frac{\gamma}{16}\left\|A^{2 \alpha} u\right\|^{2}+\frac{\eta}{12}\left\|A^{2 \beta} v\right\|^{2}+C_{9},
\end{aligned}
$$

Here 


$$
\begin{aligned}
& C_{9} \geq \frac{8 \beta-4-n}{8 \beta}\left(\frac{3(4+n)}{2 \beta \eta}\right)^{-\frac{(8 \beta)^{2}}{(4+n)(8 \beta-4-n)}}\left(\frac{4 C_{8}^{2}}{\gamma}\right)^{\frac{8 \beta}{8 \beta-4-n}}, \\
& \left|\left(u \nabla v, A^{2 \beta} v\right)\right| \leq\|u\|_{L^{4}}\|\nabla v\|_{L^{4}}\left\|A^{2 \beta} v\right\| \leq\|\Delta u\| \frac{n}{16 \alpha}\|u\|^{1-\frac{n}{16 \alpha}}\|\Delta v\| \frac{4+n}{16 \beta}\|v\|^{1-\frac{4+n}{16 \beta}}\left\|A^{2 \beta} v\right\| \\
& \leq C_{10}\|\Delta u\|^{\frac{n}{16 \alpha}}\|\Delta v\|^{\frac{4+n}{16 \beta}}\left\|A^{2 \beta} v\right\| \leq C_{11}\left\|A^{2 \alpha} u\right\|^{\frac{n}{16 \alpha}}\left\|A^{2 \beta} v\right\|^{1+\frac{4+n}{16 \beta}} \\
& \leq \frac{\gamma}{16}\left\|A^{2 \alpha} u\right\|^{2}+C_{12}\left\|A^{2 \beta} v\right\|^{\frac{16 \beta+4+n}{16 \beta} 32 \alpha-n} \leq \frac{\gamma}{16}\left\|A^{2 \alpha} u\right\|^{2}+\frac{\eta}{12}\left\|A^{2 \beta} v\right\|^{2}+C_{13} \text {, }
\end{aligned}
$$

Here

$$
\begin{aligned}
& C_{12} \geq \frac{32 \alpha-n}{32 \alpha} C_{11}^{\frac{32 \alpha}{32 \alpha-n}}\left(\frac{n}{2 \gamma \alpha}\right)^{-\frac{(32 \alpha)^{2}}{n(32 \alpha-n)}} \\
& C_{13} \geq \frac{(32 \alpha-n) \beta}{16 \beta \alpha-\beta n-\alpha(4+n)} C_{12} \frac{16 \beta \alpha-\beta n-\alpha(4+n)}{(32 \alpha-n) \beta}\left(\frac{12 \alpha(16 \beta+4+n)}{\eta \beta(32 \alpha-n)}\right)^{-\frac{((32 \alpha-n) \beta)^{2}}{\alpha(16 \beta+4+n)(16 \beta \alpha-\beta n-\alpha(4+n))}}, \\
& \left|\left(v \nabla u, A^{2 \beta} v\right)\right| \leq\|v\|_{L^{4}}\|\nabla u\|_{L^{4}}\left\|A^{2 \beta} v\right\| \leq\|\Delta v\| \frac{n}{16 \beta}\|v\|^{1-\frac{n}{16 \beta}}\|\Delta u\|^{\frac{4+n}{16 \alpha}}\|u\|^{1-\frac{4+n}{16 \alpha}}\left\|A^{2 \beta} v\right\| \\
& \leq C_{14}\|\Delta v\|^{\frac{n}{16 \beta}}\|\Delta u\|^{\frac{4+n}{16 \alpha}}\left\|A^{2 \beta} v\right\| \leq C_{15}\left\|A^{2 \alpha} u\right\|^{\frac{4+n}{16 \alpha}}\left\|A^{2 \beta} v\right\|^{1+\frac{n}{16 \beta}}
\end{aligned}
$$

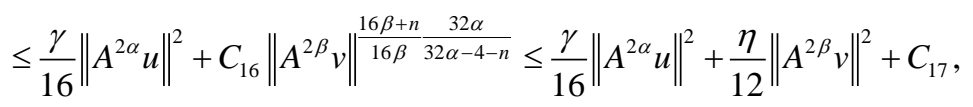

Here

$$
\begin{gathered}
C_{16} \geq \frac{32 \alpha-4-n}{32 \alpha} C_{15}^{\frac{32 \alpha-4-n}{32 \alpha}}\left(\frac{4+n}{2 \gamma \alpha}\right)^{-\frac{(32 \alpha)^{2}}{(4+n)(32 \alpha-4-n)},} \\
C_{17} \geq \frac{(32 \alpha-4-n) \beta}{16 \beta \alpha-\alpha n-\beta(4+n)} C_{16}^{\frac{16 \beta \alpha-\alpha n-\beta(4+n)}{(32 \alpha-4-n) \beta}}\left(\frac{12 \alpha(16 \beta+n)}{\eta \beta(32 \alpha-4-n)}\right)^{-\frac{((32 \alpha-4-n) \beta)^{2}}{\alpha(16 \beta+n)(16 \beta \alpha-\alpha n-\beta(4+n))},}
\end{gathered}
$$

According to the Poincare's inequalities

$$
\begin{gathered}
\left\|A^{2 \alpha} u\right\|^{2} \geq \lambda_{1}^{2 \alpha}\left\|A^{\alpha} u\right\|^{2},\left\|A^{2 \beta} v\right\|^{2} \geq \lambda_{1}^{2 \beta}\left\|A^{\beta} v\right\|^{2} \\
\left|\left(f(x), A^{2 \alpha} u\right)\right| \leq\left\|A^{\alpha} u\right\|\left\|A^{\alpha} f\right\| \leq \frac{\gamma \lambda_{1}^{2 \alpha}}{4}\left\|A^{\alpha} u\right\|^{2}+\frac{1}{\gamma \lambda_{1}^{2 \alpha}}\left\|A^{\alpha} f\right\|^{2}, \\
\left|\left(g(x), A^{2 \beta} v\right)\right| \leq\left\|A^{\beta} v\right\|\left\|A^{\beta} g\right\| \leq \frac{\eta \lambda_{1}^{2 \beta}}{4}\left\|A^{\beta} v\right\|^{2}+\frac{1}{\eta \lambda_{1}^{2 \beta}}\left\|A^{\beta} g\right\|^{2},
\end{gathered}
$$

From (2.12)-(2.17), we have

$$
\begin{aligned}
& \frac{1}{2} \frac{\mathrm{d}}{\mathrm{d} t}\left(\left\|A^{\alpha} u\right\|^{2}+\left\|A^{\beta} v\right\|^{2}\right)+\frac{\gamma}{4}\left\|A^{2 \alpha} u\right\|^{2}+\frac{\gamma \lambda_{1}^{2 \alpha}}{2}\left\|A^{\alpha} u\right\|^{2}+\frac{\eta}{4}\left\|A^{2 \beta} v\right\|^{2}+\frac{\eta \lambda_{1}^{2 \beta}}{2}\left\|A^{\beta} v\right\|^{2} \\
& \leq \frac{\gamma \lambda_{1}^{2 \alpha}}{4}\left\|A^{\alpha} u\right\|^{2}+\frac{1}{\gamma \lambda_{1}^{2 \alpha}}\left\|A^{\alpha} f\right\|^{2}+\frac{\eta \lambda_{1}^{2 \beta}}{4}\left\|A^{\beta} v\right\|^{2}+\frac{1}{\eta \lambda_{1}^{2 \beta}}\left\|A^{\beta} g\right\|^{2}+C_{18},
\end{aligned}
$$

Here 


$$
C_{18} \geq C_{6}+C_{9}+C_{13}+C_{17}
$$

So

$$
\frac{1}{2} \frac{\mathrm{d}}{\mathrm{d} t}\left(\left\|A^{\alpha} u\right\|^{2}+\left\|A^{\beta} v\right\|^{2}\right)+\frac{\gamma \lambda_{1}^{2 \alpha}}{4}\left\|A^{\alpha} u\right\|^{2}+\frac{\eta \lambda_{1}^{2 \beta}}{4}\left\|A^{\beta} v\right\|^{2} \leq \frac{1}{\gamma \lambda_{1}^{2 \alpha}}\left\|A^{\alpha} f\right\|^{2}+\frac{1}{\eta \lambda_{1}^{2 \beta}}\left\|A^{\beta} g\right\|^{2}+C_{18} .
$$

We obtain

$$
\frac{\mathrm{d}}{\mathrm{d} t}\left(\left\|A^{\alpha} u\right\|^{2}+\left\|A^{\beta} v\right\|^{2}\right)+a\left(\left\|A^{\alpha} u\right\|^{2}+\left\|A^{\beta} v\right\|^{2}\right) \leq \frac{1}{a}\left(\left\|A^{\alpha} f\right\|^{2}+\left\|A^{\beta} g\right\|^{2}\right)+2 C_{18} .
$$

Using the Gronwall's inequality, the Lemma 2 is proved.

\section{Global Attractor and Dimension Estimation}

Theorem 1. Assume that $(f(x), g(x)) \in L^{2}(\Omega) \times L^{2}(\Omega)$ and $\left(u_{0}, v_{0}\right) \in H^{2 \alpha}(\Omega) \times H^{2 \beta}(\Omega)$, so problem (1.1) exist a unique solution $w(u(x, t), v(x, t)) \in L^{2}\left(0, \infty ; H^{2 \alpha}(\Omega) \times H^{2 \beta}(\Omega)\right)$.

Proof. By the method of Galerkin and Lemma 1-Lemma 2,we can easily obtain the existence of solutions. Next, we prove the uniqueness of solutions in detail.

Assume $w_{1}\left(u_{1}, v_{1}\right), w_{2}\left(u_{2}, v_{2}\right)$ are two solutions of problem (1.1), let $w(u, v)=w_{1}\left(u_{1}, v_{1}\right)-w_{2}\left(u_{2}, v_{2}\right)$, Here $u=u_{1}-u_{2}, v=v_{1}-v_{2}$, so the difference of the two solution satisfies

$$
\begin{aligned}
& \left\{\begin{array}{l}
\frac{\partial u_{1}}{\partial t}+\left(u_{1} \cdot \nabla\right) u_{1}-\left(v_{1} \cdot \nabla\right) v_{1}+\gamma A^{2 \alpha} u_{1}=f(x), \\
\frac{\partial v_{1}}{\partial t}+\left(u_{1} \cdot \nabla\right) v_{1}-\left(v_{1} \cdot \nabla\right) u_{1}+\eta A^{2 \beta} v_{1}=g(x), \\
\nabla u_{1}=\nabla v_{1}=0, \\
\left(u_{1}, v_{1}\right)(x, 0)=\left(u_{10}, v_{10}\right)(x), \\
\left.u_{1}(x, t)\right|_{\partial \Omega}=\left.v_{1}(x, t)\right|_{\partial \Omega}=0 .
\end{array}\right. \\
& \left\{\begin{array}{l}
\frac{\partial u_{2}}{\partial t}+\left(u_{2} \cdot \nabla\right) u_{2}-\left(v_{2} \cdot \nabla\right) v_{2}+\gamma A^{2 \alpha} u_{2}=f(x), \\
\frac{\partial v_{2}}{\partial t}+\left(u_{2} \cdot \nabla\right) v_{2}-\left(v_{2} \cdot \nabla\right) u_{2}+\eta A^{2 \beta} v_{2}=g(x), \\
\nabla u_{2}=\nabla v_{2}=0, \\
\left(u_{2}, v_{2}\right)(x, 0)=\left(u_{20}, v_{20}\right)(x), \\
\left.u_{2}(x, t)\right|_{\partial \Omega}=\left.v_{2}(x, t)\right|_{\partial \Omega}=0 .
\end{array}\right.
\end{aligned}
$$

The two above formulae subtract and obtain

$$
\left\{\begin{array}{l}
\frac{\partial u}{\partial t}+u \nabla u_{1}+u_{2} \nabla u-v \nabla v_{1}-v_{2} \nabla v+\gamma A^{2 \alpha} u=0 \\
\frac{\partial v}{\partial t}+u \nabla v_{1}+u_{2} \nabla v-v \nabla u_{1}-v_{2} \nabla u+\eta A^{2 \beta} v=0 \\
\nabla u=\nabla v=0 \\
(u, v)(x, 0)=\left(u_{0}, v_{0}\right)(x) \\
\left.u(x, t)\right|_{\partial \Omega}=\left.v(x, t)\right|_{\partial \Omega}=0 .
\end{array}\right.
$$

For the problem (3.3) multiply the first equation by $u$ with both sides and obtain

$$
\frac{1}{2} \frac{\mathrm{d}}{\mathrm{d} t}\|u\|^{2}+\left(u \nabla u_{1}+u_{2} \nabla u-v \nabla v_{1}-v_{2} \nabla v, u\right)+\gamma\left\|A^{\alpha} u\right\|^{2}=0,
$$

For the problem (3.3) multiply the second equation by $v$ with both sides and obtain 


$$
\frac{1}{2} \frac{\mathrm{d}}{\mathrm{d} t}\|v\|^{2}+\left(u \nabla v_{1}+u_{2} \nabla v-v \nabla u_{1}-v_{2} \nabla u, v\right)+\eta\left\|A^{\beta} v\right\|^{2}=0,
$$

According to

$$
b\left(u_{2}, u, u\right)=b\left(u_{2}, v, v\right)=0, b\left(v_{2}, v, u\right)=-b\left(v_{2}, u, v\right) .
$$

According to (3.1) + (3.2), we have

$$
\frac{1}{2} \frac{\mathrm{d}}{\mathrm{d} t}\left(\|u\|^{2}+\|v\|^{2}\right)+\left(u \nabla u_{1}-v \nabla v_{1}, u\right)+\left(u \nabla v_{1}-v \nabla u_{1}, v\right)+\gamma\left\|A^{\alpha} u\right\|^{2}+\eta\left\|A^{\beta} v\right\|^{2}=0,
$$

According to Sobolev inequality, when $n<4$

$$
\begin{gathered}
\|u\|_{\infty} \leq C_{19}\|\Delta u\|^{\frac{n}{4}}\|u\|^{\frac{4-n}{4}} \leq C_{20}\|\Delta u\| \leq C_{20} \lambda_{1}^{\alpha-1}\left\|A^{\alpha} u\right\|, \\
\|v\|_{\infty} \leq C_{21}\|\Delta v\|^{\frac{n}{4}}\|v\|^{\frac{4-n}{4}} \leq C_{22}\|\Delta v\| \leq C_{22} \lambda_{1}^{\beta-1}\left\|A^{\beta} v\right\|,
\end{gathered}
$$

According to (3.8)-(3.9),we can get

$$
\begin{aligned}
& \left|\left(u \nabla u_{1}, u\right)\right| \leq\|u\|\|\nabla u\|\left\|u_{1}\right\|_{\infty} \leq C_{23}\|u\|\left\|A^{\alpha} u\right\| \leq \frac{\gamma}{12}\left\|A^{\alpha} u\right\|^{2}+\frac{3 C_{23}^{2}}{\gamma}\|u\|^{2}, \\
& \left|\left(v \nabla v_{1}, u\right)\right| \leq\|v\|\|\nabla u\|\left\|v_{1}\right\|_{\infty} \leq C_{24}\|v\|\left\|A^{\alpha} u\right\| \leq \frac{\gamma}{12}\left\|A^{\alpha} u\right\|^{2}+\frac{3 C_{24}^{2}}{\gamma}\|v\|^{2}, \\
& \left|\left(v \nabla u_{1}, v\right)\right| \leq\|v\|\|\nabla v\|\left\|u_{1}\right\|_{\infty} \leq C_{25}\|v\|\left\|A^{\beta} v\right\| \leq \frac{\eta}{12}\left\|A^{\beta} v\right\|^{2}+\frac{3 C_{25}^{2}}{\eta}\|v\|^{2}, \\
& \left|\left(u \nabla v_{1}, v\right)\right| \leq\|u\|\|\nabla v\|\left\|v_{1}\right\|_{\infty} \leq C_{26}\|u\|\left\|A^{\beta} v\right\| \leq \frac{\eta}{12}\left\|A^{\beta} v\right\|^{2}+\frac{3 C_{26}^{2}}{\eta}\|u\|^{2},
\end{aligned}
$$

From (3.10)-(3.13),

$$
\begin{aligned}
& \frac{1}{2} \frac{\mathrm{d}}{\mathrm{d} t}\left(\|u\|^{2}+\|v\|^{2}\right)+\frac{\gamma}{2}\left\|A^{\alpha} u\right\|^{2}+\frac{\gamma \lambda_{1}^{2 \alpha}}{2}\|u\|^{2}+\frac{\eta}{2}\left\|A^{\beta} v\right\|^{2}+\frac{\eta \lambda_{1}^{2 \beta}}{2}\|v\|^{2} \\
& \leq \frac{\gamma}{4}\left\|A^{\alpha} u\right\|^{2}+\frac{\eta}{4}\left\|A^{\beta} v\right\|^{2}+\gamma \lambda_{1}^{2 \alpha}\|u\|^{2}+\eta \lambda_{1}^{2 \beta}\|v\|^{2} .
\end{aligned}
$$

Here $\gamma \lambda_{1}^{2 \alpha} \geq \frac{3 C_{23}^{2}}{\gamma}+\frac{3 C_{26}^{2}}{\eta}, \eta \lambda_{1}^{2 \beta} \geq \frac{3 C_{24}^{2}}{\gamma}+\frac{3 C_{25}^{2}}{\eta}$.

So, we have

$$
\begin{gathered}
\frac{1}{2} \frac{\mathrm{d}}{\mathrm{d} t}\left(\|u\|^{2}+\|v\|^{2}\right)+\frac{\gamma}{4}\left\|A^{\alpha} u\right\|^{2}+\frac{\eta}{4}\left\|A^{\beta} v\right\|^{2} \leq \frac{\gamma \lambda_{1}^{2 \alpha}}{2}\|u\|^{2}+\frac{\eta \lambda_{1}^{2 \beta}}{2}\|v\|^{2} . \\
\frac{\mathrm{d}}{\mathrm{d} t}\left(\|u\|^{2}+\|v\|^{2}\right) \leq \gamma \lambda_{1}^{2 \alpha}\|u\|^{2}+\eta \lambda_{1}^{2 \beta}\|v\|^{2} .
\end{gathered}
$$

Let $b=\max \left\{\gamma \lambda_{1}^{2 \alpha}, \eta \lambda_{1}^{2 \beta}\right\}$, so we obtain

$$
\frac{\mathrm{d}}{\mathrm{d} t}\left(\|u\|^{2}+\|v\|^{2}\right) \leq b\left(\|u\|^{2}+\|v\|^{2}\right)
$$

According to the consistent Gronwall inequality,

$$
\left(\|u\|^{2}+\|v\|^{2}\right)^{2} \leq\left(\left\|u_{0}\right\|^{2}+\left\|v_{0}\right\|^{2}\right) \mathrm{e}^{b t}=0 .
$$

So we can get $u=v=0$, the uniqueness is proved.

Theorem 2. [9] Let $E$ be a Banach space, and $\{S(t)\}(t \geq 0)$ are the semigroup operators on $E$. 
$S(t): E \rightarrow E, S(t) \cdot S(\tau)=S(t+\tau), S(0)=I$, here $I$ is a unit operator. Set $S(t)$ satisfy the follow conditions

1) $S(t)$ is bounded. Namely $\forall R>0,\|u\|_{\infty} \leq R$, it exists a constant $C(R)$, so that

$\|S(t) u\|_{E} \leq C(R)(t \in[0,+\infty))$;

2) It exists a bounded absorbing set $B_{0} \subset E$, namely $\forall B \subset E$, it exists a constant $t_{0}$, so that $S(t) B \subset B_{0}\left(t>t_{0}\right)$;

3) When $t>0, S(t)$ is a completely continuous operator $A$.

Therefore, the semigroup operators $S(t)$ exist a compact global attractor.

Theorem 3. Assume $\left(u_{0}, v_{0}\right) \in E=H^{2 \alpha}(\Omega) \times H^{2 \beta}(\Omega), \quad(f(x), g(x)) \in L^{2}(\Omega) \times L^{2}(\Omega), \quad \alpha>\frac{n}{2}, \quad \beta>\frac{n}{2}$.

Problem (1.1) have global attractor $A=w\left(B_{0}\right)=\bigcap_{s \geq 0} S(t) B_{0}$.

Proof.

1) When $\left\|u_{0}\right\|_{H^{2 \alpha}(\Omega)}+\left\|v_{0}\right\|_{H^{2 \beta}(\Omega)} \leq R_{1}+R_{2}$. From Lemma 1,

$$
\left\|S(t) u_{0}\right\|_{H^{2 \alpha}(\Omega)}+\left\|S(t) v_{0}\right\|_{H^{2 \beta}(\Omega)}=\|u\|_{H^{2 \alpha}(\Omega)}+\|v\|_{H^{2 \beta}(\Omega)} \leq C_{27}\left(\left\|u_{0}\right\|_{H^{2 \alpha}(\Omega)}+\left\|v_{0}\right\|_{H^{2 \beta}(\Omega)}\right) \leq C_{27}\left(R_{1}+R_{2}\right) .
$$

So $S(t)$ in $E$ is uniformly bounded.

2) $(u(t), v(t))=S(t)\left(u_{0}, v_{0}\right)$ has $E$ in a bounded absorbing set

$$
B_{0}=\left\{(u, v) \in E:\left\|u_{0}\right\|_{H^{2 \alpha}(\Omega)}+\left\|v_{0}\right\|_{H^{2 \beta}(\Omega)} \leq R_{1}+R_{2}\right\} .
$$

From Lemma 2, when $\left\|u_{0}\right\|_{H^{2 \alpha}(\Omega)} \leq R_{1},\left\|v_{0}\right\|_{H^{2 \beta}(\Omega)} \leq R_{2}$, there is

$$
\left\|A^{2 \alpha} u\right\|+\left\|A^{2 \beta} v\right\|=\|u\|_{H^{2 \alpha}(\Omega)}+\|v\|_{H^{2 \beta}(\Omega)} \leq C_{28}\left(\left\|u_{0}\right\|_{H^{2 \alpha}(\Omega)}+\left\|v_{0}\right\|_{H^{2 \beta}(\Omega)}\right) \leq C_{28}\left(R_{1}+R_{2}\right) .
$$

Since $E \rightarrow E$ is tightly embedded, so $B_{0}$ is $S(t)$ in the tight absorbing set in $E$.

3) So the semigroup operator $S(t): E \rightarrow E$ is completely continuous.

In order to estimate the Hausdorff and fractal dimension of the global attractor A of problem (1.1), let problem (1.1) linearize and obtain

$$
\left\{\begin{array}{l}
\frac{\partial U}{\partial t}+(U \cdot \nabla) u+(u \cdot \nabla) U-(V \cdot \nabla) v-(v \cdot \nabla) V+\gamma A^{2 \alpha} U=0, \\
\frac{\partial V}{\partial t}+(U \cdot \nabla) v+(u \cdot \nabla) V-(V \cdot \nabla) u-(v \cdot \nabla) U+\eta A^{2 \beta} V=0, \\
U(0)=U_{0}, V(0)=V_{0} .
\end{array}\right.
$$

Assume $\left(U_{0}, V_{0}\right) \in H^{2 \alpha}(\Omega) \times H^{2 \beta}(\Omega), \quad(U(t), V(t))$ is the solutions of the problem (3.14). We know $(u, v) \in L^{\infty}\left(0, \infty ; H^{2 \alpha}(\Omega) \times H^{2 \beta}(\Omega)\right)$. It is easy to prove the problem (3.14) has the uniqueness of solutions $(U(t), V(t)) \in L^{\infty}\left(0, \infty ; H^{2 \alpha}\left(\Omega \times H^{2 \beta}(\Omega)\right)\right)$.

To prove $S(t)$ in $\left(u_{0}, v_{0}\right)$ has differential, let $(u(t), v(t))=S(t)\left(u_{0}, v_{0}\right)$, so there has

$$
\left(D S(t)\left(u_{0}, v_{0}\right)\right)\left(U_{0}, V_{0}\right)=(U(t), V(t)) .
$$

Theorem 4. Assume $R_{3}, R_{4}, R_{5}, R_{6}$ and $T$ are constants, so it exists a constant $C_{23}=C_{29}\left(R_{3}, R_{4}, R_{5}, R_{6}, T\right)$, and $\forall u_{0}, u_{0}^{\prime}, v_{0}, v_{0}^{\prime}, t$ has $\left\|u_{0}\right\|_{H^{2 \alpha}(\Omega)} \leq R_{3}, \quad\left\|u_{0}^{\prime}\right\|_{H^{2 \alpha}(\Omega)} \leq R_{4}, \quad\left\|v_{0}\right\|_{H^{2 \beta}(\Omega)} \leq R_{5}, \quad\left\|v_{0}^{\prime}\right\|_{H^{2 \beta}(\Omega)} \leq R_{6},\|t\| \leq T$, so there is

$$
\begin{aligned}
& \left\|S(t)\left(u_{0}+u_{0}^{\prime}, v_{0}+v_{0}^{\prime}\right)-S(t)\left(u_{0}, v_{0}\right)-\left(D S(t)\left(u_{0}, v_{0}\right)\right)\left(u_{0}^{\prime}, v_{0}^{\prime}\right)\right\|_{H^{2 \alpha}(\Omega) \times H^{2 \beta}(\Omega)}^{2} \\
& \leq C_{29}\left(\left\|u_{0}^{\prime}\right\|_{H^{2 \alpha}(\Omega)}^{2}+\left\|v_{0}^{\prime}\right\|_{H^{2 \beta}(\Omega)}^{2}\right) .
\end{aligned}
$$

Proof. Meet the initial value problem (3.14) of respectively for $\left(u_{0}, v_{0}\right),\left(u_{0}+u_{0}^{\prime}, v_{0}+v_{0}^{\prime}\right)$ solutions for $(u, v),\left(u_{1}, v_{1}\right)$, let $\theta_{1}=u_{1}-u, \theta_{2}=v_{1}-v$. So $\theta_{1}, \theta_{2}$ satisfies 


$$
\left\{\begin{array}{l}
\frac{\partial \theta_{1}}{\partial t}+u_{1} \nabla u_{1}-u \nabla u-v_{1} \nabla v_{1}+v \nabla v+\gamma A^{2 \alpha} \theta_{1}=0 \\
\frac{\partial \theta_{2}}{\partial t}+u_{1} \nabla v_{1}-u \nabla v-v_{1} \nabla u_{1}+v \nabla u+\eta A^{2 \beta} \theta_{2}=0 \\
\theta_{1}(x, 0)=u_{0}^{\prime}, \theta_{2}(x, 0)=v_{0}^{\prime} .
\end{array}\right.
$$

Here

$$
\begin{aligned}
& u_{1} \nabla u_{1}-u \nabla u-v_{1} \nabla v_{1}+v \nabla v=\theta_{1} \nabla u_{1}+u \nabla \theta_{1}-\theta_{2} \nabla v_{1}-v \nabla \theta_{2}, \\
& u_{1} \nabla v_{1}-u \nabla v-v_{1} \nabla u_{1}+v \nabla u=\theta_{1} \nabla v_{1}+u \nabla \theta_{2}-\theta_{2} \nabla u_{1}-v \nabla \theta_{1},
\end{aligned}
$$

For the problem (3.16) multiply the first equation by $\theta_{1}$ with both sides and for the problem (3.16) multiply the second equation by $\theta_{2}$ with both sides and obtain

$$
\left\{\begin{array}{l}
\frac{1}{2} \frac{\mathrm{d}}{\mathrm{d} t}\left\|\theta_{1}\right\|^{2}+\left(\theta_{1} \nabla u_{1}+u \nabla \theta_{1}-\theta_{2} \nabla v_{1}-v \nabla \theta_{2}, \theta_{1}\right)+\gamma\left\|A^{\alpha} \theta_{1}\right\|^{2}=0, \\
\frac{1}{2} \frac{\mathrm{d}}{\mathrm{d} t}\left\|\theta_{2}\right\|^{2}+\left(\theta_{1} \nabla v_{1}+u \nabla \theta_{2}-\theta_{2} \nabla u_{1}-v \nabla \theta_{1}, \theta_{2}\right)+\eta\left\|A^{\beta} \theta_{2}\right\|^{2}=0,
\end{array}\right.
$$

Then

$$
\frac{\mathrm{d}}{\mathrm{d} t}\left(\left\|\theta_{1}\right\|^{2}+\left\|\theta_{2}\right\|^{2}\right) \leq 2 a\left(\left\|\theta_{1}\right\|^{2}+\left\|\theta_{2}\right\|^{2}\right)
$$

Here $a=\min \left\{\frac{\gamma \lambda_{1}^{2 \alpha}}{2}, \frac{\eta \lambda_{1}^{2 \beta}}{2}\right\}$.

For the problem (3.16) multiply the first equation by $A^{2 \alpha} \theta_{1}$ with both sides and for the problem (3.16) multiply the second equation by $A^{2 \beta} \theta_{2}$ with both sides and obtain

$$
\left\{\begin{array}{l}
\frac{1}{2} \frac{\mathrm{d}}{\mathrm{d} t}\left\|A^{\alpha} \theta_{1}\right\|^{2}+\left(\theta_{1} \nabla u_{1}+u \nabla \theta_{1}-\theta_{2} \nabla v_{1}-v \nabla \theta_{2}, A^{2 \alpha} \theta_{1}\right)+\gamma\left\|A^{2 \alpha} \theta_{1}\right\|^{2}=0, \\
\frac{1}{2} \frac{\mathrm{d}}{\mathrm{d} t}\left\|A^{\beta} \theta_{2}\right\|^{2}+\left(\theta_{1} \nabla v_{1}+u \nabla \theta_{2}-\theta_{2} \nabla u_{1}-v \nabla \theta_{1}, A^{2 \beta} \theta_{2}\right)+\eta\left\|A^{2 \beta} \theta_{2}\right\|^{2}=0,
\end{array}\right.
$$

According to the Sobolev's interpolation inequalities

$$
\begin{aligned}
& \|\nabla u\|_{\infty} \leq C_{30}\left\|A^{\alpha} u\right\|^{\frac{2+n}{4 \alpha}}\|u\|^{\frac{4 \alpha-2-n}{4 \alpha}}, \\
& \|\nabla v\|_{\infty} \leq C_{31}\left\|A^{\beta} v\right\|^{\frac{2+n}{4 \beta}}\|v\|^{\frac{4 \beta-2-n}{4 \beta}},
\end{aligned}
$$

According to (3.22)-(3.23), we have

$$
\begin{aligned}
\left|\left(\theta_{1} \nabla u_{1}, A^{2 \alpha} \theta_{1}\right)\right| & \leq\left\|\theta_{1}\right\|\left\|\nabla u_{1}\right\| \infty\left\|A^{2 \alpha} \theta_{1}\right\| \leq C_{30}\left\|\theta_{1}\right\|\left\|A^{\alpha} u_{1}\right\|^{\frac{2+n}{4 \alpha}}\left\|u_{1}\right\|^{\frac{4 \alpha-2-n}{4 \alpha}}\left\|A^{2 \alpha} \theta_{1}\right\| \\
& \leq C_{32}\left\|\theta_{1}\right\|\left\|A^{2 \alpha} \theta_{1}\right\| \leq \frac{\gamma}{8}\left\|A^{2 \alpha} \theta_{1}\right\|^{2}+\frac{2 C_{32}^{2}}{\gamma}\left\|\theta_{1}\right\|^{2},
\end{aligned}
$$

In a similar way, we can obtain

$$
\begin{aligned}
\left|\left(u \nabla \theta_{1}, A^{2 \alpha} \theta_{1}\right)\right| & \leq\left\|\theta_{1}\right\|\|\nabla u\|_{\infty}\left\|A^{2 \alpha} \theta_{1}\right\| \leq C_{30}\left\|\theta_{1}\right\|\left\|A^{\alpha} u\right\|^{\frac{2+n}{4 \alpha}}\|u\|^{\frac{4 \alpha-2-n}{4 \alpha}}\left\|A^{2 \alpha} \theta_{1}\right\| \\
& \leq C_{33}\left\|\theta_{1}\right\|\left\|A^{2 \alpha} \theta_{1}\right\| \leq \frac{\gamma}{8}\left\|A^{2 \alpha} \theta_{1}\right\|^{2}+\frac{2 C_{33}^{2}}{\gamma}\left\|\theta_{1}\right\|^{2},
\end{aligned}
$$




$$
\begin{aligned}
& \left|\left(\theta_{2} \nabla v_{1}, A^{2 \alpha} \theta_{1}\right)\right| \leq\left\|\theta_{2}\right\|\left\|\nabla v_{1}\right\|_{\infty}\left\|A^{2 \alpha} \theta_{1}\right\| \leq C_{31}\left\|\theta_{2}\right\|\left\|A^{\beta} v_{1}\right\|^{\frac{2+n}{4 \beta}}\left\|v_{1}\right\|^{\frac{4 \beta-2-n}{4 \beta}}\left\|A^{2 \alpha} \theta_{1}\right\| \\
& \leq C_{34}\left\|\theta_{2}\right\|\left\|A^{2 \alpha} \theta_{1}\right\| \leq \frac{\gamma}{8}\left\|A^{2 \alpha} \theta_{1}\right\|^{2}+\frac{2 C_{34}^{2}}{\gamma}\left\|\theta_{2}\right\|^{2}, \\
& \left|\left(v \nabla \theta_{2}, A^{2 \alpha} \theta_{1}\right)\right| \leq\left\|\theta_{2}\right\|\|\nabla v\|\left\|A^{2 \alpha} \theta_{1}\right\| \leq C_{31}\left\|\theta_{2}\right\|\left\|A^{\beta} v\right\|^{\frac{2+n}{4 \beta}}\|v\|^{\frac{4 \beta-2-n}{4 \beta}}\left\|A^{2 \alpha} \theta_{1}\right\| \\
& \leq C_{35}\left\|\theta_{2}\right\|\left\|A^{2 \alpha} \theta_{1}\right\| \leq \frac{\gamma}{8}\left\|A^{2 \alpha} \theta_{1}\right\|^{2}+\frac{2 C_{35}^{2}}{\gamma}\left\|\theta_{2}\right\|^{2}, \\
& \left|\left(\theta_{1} \nabla v_{1}, A^{2 \beta} \theta_{2}\right)\right| \leq\left\|\theta_{1}\right\|\left\|\nabla v_{1}\right\|_{\infty}\left\|A^{2 \beta} \theta_{2}\right\| \leq C_{34}\left\|\theta_{1}\right\|\left\|A^{2 \beta} \theta_{2}\right\| \leq \frac{\eta}{8}\left\|A^{2 \beta} \theta_{2}\right\|^{2}+\frac{2 C_{34}^{2}}{\eta}\left\|\theta_{1}\right\|^{2}, \\
& \left|\left(u \nabla \theta_{2}, A^{2 \beta} \theta_{2}\right)\right| \leq\left\|\theta_{2}\right\|\|\nabla u\|_{\infty}\left\|A^{2 \beta} \theta_{2}\right\| \leq C_{33}\left\|\theta_{2}\right\|\left\|A^{2 \beta} \theta_{2}\right\| \leq \frac{\eta}{8}\left\|A^{2 \beta} \theta_{2}\right\|^{2}+\frac{2 C_{33}^{2}}{\eta}\left\|\theta_{2}\right\|^{2}, \\
& \left|\left(\theta_{2} \nabla u_{1}, A^{2 \beta} \theta_{2}\right)\right| \leq\left\|\theta_{2}\right\|\left\|\nabla u_{1}\right\|\left\|_{\infty}\right\| A^{2 \beta} \theta_{2}\left\|\leq C_{32}\right\| \theta_{2}\|\| A^{2 \beta} \theta_{2}\left\|\leq \frac{\eta}{8}\right\| A^{2 \beta} \theta_{2}\left\|^{2}+\frac{2 C_{32}^{2}}{\eta}\right\| \theta_{2} \|^{2}, \\
& \left|\left(v \nabla \theta_{1}, A^{2 \beta} \theta_{2}\right)\right| \leq\left\|\theta_{1}\right\|\|\nabla v\|_{\infty}\left\|A^{2 \beta} \theta_{2}\right\| \leq C_{35}\left\|\theta_{1}\right\|\left\|A^{2 \beta} \theta_{2}\right\| \leq \frac{\eta}{8}\left\|A^{2 \beta} \theta_{2}\right\|^{2}+\frac{2 C_{35}^{2}}{\eta}\left\|\theta_{1}\right\|^{2},
\end{aligned}
$$

So, we can get

$$
\frac{1}{2} \frac{\mathrm{d}}{\mathrm{d} t}\left(\left\|A^{\alpha} \theta_{1}\right\|^{2}+\left\|A^{\beta} \theta_{2}\right\|^{2}\right)+\gamma\left\|A^{2 \alpha} \theta_{1}\right\|^{2}+\eta\left\|A^{2 \beta} \theta_{2}\right\|^{2} \leq \frac{\gamma}{2}\left\|A^{2 \alpha} \theta_{1}\right\|^{2}+\frac{\eta}{2}\left\|A^{2 \beta} \theta_{2}\right\|^{2}+c\left(\left\|\theta_{1}\right\|^{2}+\left\|\theta_{2}\right\|^{2}\right),
$$

Here $c=\max \left\{\frac{2 C_{32}^{2}}{\gamma}+\frac{2 C_{33}^{2}}{\gamma}+\frac{2 C_{34}^{2}}{\eta}+\frac{2 C_{35}^{2}}{\eta}, \frac{2 C_{32}^{2}}{\eta}+\frac{2 C_{33}^{2}}{\eta}+\frac{2 C_{34}^{2}}{\gamma}+\frac{2 C_{35}^{2}}{\gamma}\right\}$, we obtain

$$
\frac{\mathrm{d}}{\mathrm{d} t}\left(\left\|A^{\alpha} \theta_{1}\right\|^{2}+\left\|A^{\beta} \theta_{2}\right\|^{2}\right) \leq 2 c\left(\left\|\theta_{1}\right\|^{2}+\left\|\theta_{2}\right\|^{2}\right),
$$

According to the Poincare's inequalities

$$
\left\|\theta_{1}\right\|^{2} \leq \frac{1}{\lambda_{1}^{2 \alpha}}\left\|A^{\alpha} \theta_{1}\right\|^{2},\left\|\theta_{2}\right\|^{2} \leq \frac{1}{\lambda_{1}^{2 \beta}}\left\|A^{\beta} \theta_{2}\right\|^{2} .
$$

Let $d=\max \left\{\frac{c}{\lambda_{1}^{2 \alpha}}, \frac{c}{\lambda_{1}^{2 \beta}}\right\}$,

$$
\frac{\mathrm{d}}{\mathrm{d} t}\left(\left\|A^{\alpha} \theta_{1}\right\|^{2}+\left\|A^{\beta} \theta_{2}\right\|^{2}\right) \leq 2 d\left(\left\|A^{\alpha} \theta_{1}\right\|^{2}+\left\|A^{\beta} \theta_{2}\right\|^{2}\right),
$$

According to Gronwall's inequalities, we obtain

$$
\left(\left\|A^{\alpha} \theta_{1}\right\|^{2}+\left\|A^{\beta} \theta_{2}\right\|^{2}\right) \leq\left(\left\|A^{\alpha} u_{0}^{\prime}\right\|^{2}+\left\|A^{\beta} v_{0}^{\prime}\right\|^{2}\right) \mathrm{e}^{2 d t} .
$$

Let $(U, V)$ be the solutions of the linear Equation (3.14), and satisfies $(U(0), V(0))=\left(u_{0}^{\prime}, v_{0}^{\prime}\right)$, Assume

$$
\begin{aligned}
\left(w_{1}, w_{2}\right) & =\left(u_{1}-u-U, v_{1}-v-V\right) \\
& =S(t)\left(u_{0}+u_{0}^{\prime}, v_{0}+v_{0}^{\prime}\right)-S(t)\left(u_{0}, v_{0}\right)-\left(D S(t)\left(u_{0}, v_{0}\right)\right)\left(u_{0}^{\prime}, v_{0}^{\prime}\right),
\end{aligned}
$$

So, we can get 


$$
\left\{\begin{array}{l}
\frac{\mathrm{d}}{\mathrm{d} t} w_{1}+u_{1} \nabla u_{1}-u \nabla u-v_{1} \nabla v_{1}+v \nabla v-U \nabla u-u \nabla U+V \nabla v+v \nabla V+\gamma A^{2 \alpha} w_{1}=0 . \\
\frac{\mathrm{d}}{\mathrm{d} t} w_{2}+u_{1} \nabla v_{1}-u \nabla v-v_{1} \nabla u_{1}+v \nabla u-U \nabla v-u \nabla V+V \nabla u+v \nabla U+\eta A^{2 \beta} w_{2}=0 . \\
w_{1}(x, 0)=0, w_{2}(x, 0)=0
\end{array}\right.
$$

Here

$$
\begin{aligned}
& u_{1} \nabla u_{1}-u \nabla u-v_{1} \nabla v_{1}+v \nabla v-U \nabla u-u \nabla U+V \nabla v+v \nabla V \\
& =\theta_{1} \nabla \theta_{1}+w_{1} \nabla u+u \nabla w_{1}-\theta_{2} \nabla \theta_{2}-w_{2} \nabla v-v \nabla w_{2} \\
& u_{1} \nabla v_{1}-u \nabla v-v_{1} \nabla u_{1}+v \nabla u-U \nabla v-u \nabla V+V \nabla u+v \nabla U \\
& =\theta_{1} \nabla \theta_{2}+w_{1} \nabla v+u \nabla w_{2}-\theta_{2} \nabla \theta_{1}-w_{2} \nabla u-v \nabla w_{1} .
\end{aligned}
$$

For the problem (3.33) multiply the first equation by $w_{1}$ with both sides and for the problem (3.33) multiply the second equation by $w_{2}$ with both sides and obtain

$$
\left\{\begin{array}{l}
\frac{1}{2} \frac{\mathrm{d}}{\mathrm{d} t}\left\|w_{1}\right\|^{2}+\left(\theta_{1} \nabla \theta_{1}+w_{1} \nabla u+u \nabla w_{1}-\theta_{2} \nabla \theta_{2}-w_{2} \nabla v-v \nabla w_{2}, w_{1}\right)+\gamma\left\|A^{\alpha} w_{1}\right\|^{2}=0 \\
\frac{1}{2} \frac{\mathrm{d}}{\mathrm{d} t}\left\|w_{2}\right\|^{2}+\left(\theta_{1} \nabla \theta_{2}+w_{1} \nabla v+u \nabla w_{2}-\theta_{2} \nabla \theta_{1}-w_{2} \nabla u-v \nabla w_{1}, w_{2}\right)+\eta\left\|A^{\beta} w_{2}\right\|^{2}=0
\end{array}\right.
$$

According to (3.8)-(3.9), then

$$
\begin{aligned}
& \left|\left(\theta_{1} \nabla \theta_{1}, w_{1}\right)\right| \leq\left\|\theta_{1}\right\|\left\|\nabla \theta_{1}\right\|\left\|w_{1}\right\|_{\infty} \leq C_{36}\left\|A^{\alpha} \theta_{1}\right\|\left\|A^{\alpha} w_{1}\right\| \leq \frac{\gamma}{10}\left\|A^{\alpha} w_{1}\right\|^{2}+\frac{5 C_{36}^{2}}{2 \gamma}\left\|A^{\alpha} \theta_{1}\right\|^{2} . \\
& \left|\left(w_{1} \nabla u, w_{1}\right)\right| \leq\left\|w_{1}\right\|\left\|\nabla w_{1}\right\|\|u\|_{\infty} \leq C_{37}\left\|w_{1}\right\|\left\|A^{\alpha} w_{1}\right\| \leq \frac{\gamma}{10}\left\|A^{\alpha} w_{1}\right\|^{2}+\frac{5 C_{37}^{2}}{2 \gamma}\left\|w_{1}\right\|^{2} . \\
& \left|\left(\theta_{2} \nabla \theta_{2}, w_{1}\right)\right| \leq\left\|\theta_{2}\right\|\left\|\nabla \theta_{2}\right\|\left\|w_{1}\right\|_{\infty} \leq C_{38}\left\|A^{\beta} \theta_{2}\right\|\left\|A^{\alpha} w_{1}\right\| \leq\left.\frac{\gamma}{10}\left\|A^{\alpha} w_{1}\right\|\right|^{2}+\frac{5 C_{38}^{2}}{2 \gamma}\left\|A^{\beta} \theta_{2}\right\|^{2} . \\
& \left|\left(w_{2} \nabla v, w_{1}\right)\right| \leq\left\|w_{2}\right\|\left\|\nabla w_{1}\right\|\|v\|_{\infty} \leq C_{39}\left\|w_{2}\right\|\left\|A^{\alpha} w_{1}\right\| \leq \frac{\gamma}{10}\left\|A^{\alpha} w_{1}\right\|^{2}+\frac{5 C_{39}^{2}}{2 \gamma}\left\|w_{2}\right\|^{2} . \\
& \left|\left(v \nabla w_{2}, w_{1}\right)\right| \leq\left\|w_{1}\right\|\left\|\nabla w_{2}\right\|\|v\|_{\infty} \leq C_{40}\left\|w_{1}\right\|\left\|A^{\beta} w_{2}\right\| \leq \frac{\eta}{10}\left\|A^{\beta} w_{2}\right\|^{2}+\frac{5 C_{40}^{2}}{2 \eta}\left\|w_{1}\right\|^{2} . \\
& \left|\left(\theta_{1} \nabla \theta_{2}, w_{2}\right)\right| \leq\left\|\theta_{1}\right\|\left\|\nabla \theta_{2}\right\|\left\|w_{2}\right\|_{\infty} \leq C_{41}\left\|A^{\beta} \theta_{2}\right\|\left\|A^{\beta} w_{2}\right\| \leq \frac{\eta}{10}\left\|A^{\beta} w_{2}\right\|^{2}+\frac{5 C_{41}^{2}}{2 \eta}\left\|A^{\beta} \theta_{2}\right\|^{2} . \\
& \left|\left(w_{1} \nabla v, w_{2}\right)\right| \leq\left\|w_{1}\right\|\left\|\nabla w_{2}\right\|\|v\|_{\infty} \leq C_{42}\left\|w_{1}\right\|\left\|A^{\beta} w_{2}\right\| \leq \frac{\eta}{10}\left\|A^{\beta} w_{2}\right\|^{2}+\frac{5 C_{42}^{2}}{2 \eta}\left\|w_{1}\right\|^{2} . \\
& \left|\left(\theta_{2} \nabla \theta_{1}, w_{2}\right)\right| \leq\left\|\theta_{2}\right\|\left\|\nabla \theta_{1}\right\|\left\|w_{2}\right\|_{\infty} \leq C_{43}\left\|A^{\alpha} \theta_{1}\right\|\left\|A^{\beta} w_{2}\right\| \leq \frac{\eta}{10}\left\|A^{\beta} w_{2}\right\|^{2}+\frac{5 C_{43}^{2}}{2 \eta}\left\|A^{\alpha} \theta_{1}\right\|^{2} . \\
& \left|\left(w_{2} \nabla u, w_{2}\right)\right| \leq\left\|w_{2} \mid\right\| \nabla w_{2}\|\| u\left\|_{\infty} \leq C_{44}\right\| w_{2}\|\| A^{\beta} w_{2}\left\|\leq \frac{\eta}{10}\right\| A^{\beta} w_{2}\left\|^{2}+\frac{5 C_{44}^{2}}{2 \eta}\right\| w_{2} \|^{2} . \\
& \left|\left(v \nabla w_{1}, w_{2}\right)\right| \leq\left\|w_{2}\right\|\left\|\nabla w_{1}\right\|\|v\|_{\infty} \leq C_{45}\left\|A^{\alpha} w_{1}\right\|\left\|w_{2}\right\| \leq \frac{\gamma}{10}\left\|A^{\alpha} w_{1}\right\|^{2}+\frac{5 C_{45}^{2}}{2 \gamma}\left\|w_{2}\right\|^{2} .
\end{aligned}
$$

According to, we obtain 


$$
\begin{aligned}
& \frac{1}{2} \frac{\mathrm{d}}{\mathrm{d} t}\left(\left\|w_{1}\right\|^{2}+\left\|w_{2}\right\|^{2}\right)+\gamma\left\|A^{\alpha} w_{1}\right\|^{2}+\eta\left\|A^{\beta} w_{2}\right\|^{2} \\
& \leq \frac{\gamma}{2}\left\|A^{\alpha} w_{1}\right\|^{2}+\frac{\eta}{2}\left\|A^{\beta} w_{2}\right\|^{2}+e\left(\left\|w_{1}\right\|^{2}+\left\|w_{2}\right\|^{2}\right)+k\left(\left\|\theta_{1}\right\|^{2}+\left\|\theta_{2}\right\|^{2}\right) .
\end{aligned}
$$

Here $e=\max \left\{\frac{5 C_{37}^{2}}{2 \gamma}+\frac{5 C_{40}^{2}}{2 \eta}+\frac{5 C_{42}^{2}}{2 \eta}, \frac{5 C_{39}^{2}}{2 \gamma}+\frac{5 C_{44}^{2}}{2 \gamma}+\frac{5 C_{45}^{2}}{2 \eta}\right\}, k=\max \left\{\frac{5 C_{36}^{2}}{2 \gamma}+\frac{5 C_{43}^{2}}{2 \eta}, \frac{5 C_{38}^{2}}{2 \gamma}+\frac{5 C_{41}^{2}}{2 \eta}\right\}$,

$$
\frac{1}{2} \frac{\mathrm{d}}{\mathrm{d} t}\left(\left\|w_{1}\right\|^{2}+\left\|w_{2}\right\|^{2}\right)+\frac{\gamma}{2}\left\|A^{\alpha} w_{1}\right\|^{2}+\frac{\eta}{2}\left\|A^{\beta} w_{2}\right\|^{2} \leq e\left(\left\|w_{1}\right\|^{2}+\left\|w_{2}\right\|^{2}\right)+k\left(\left\|\theta_{1}\right\|^{2}+\left\|\theta_{2}\right\|^{2}\right) .
$$

We obtain

$$
\sup _{t \in[0, T]}\left(\left\|w_{1}\right\|^{2}+\left\|w_{2}\right\|^{2}\right) \leq\left(\left\|u_{0}^{\prime}\right\|_{H^{2 \alpha}(\Omega)}^{2}+\left\|v_{0}^{\prime}\right\|_{H^{2 \beta}(\Omega)}^{2}\right) \mathrm{e}^{2 d t}
$$

So

$$
\begin{aligned}
& \left\|S(t)\left(u_{0}+u_{0}^{\prime}, v_{0}+v_{0}^{\prime}\right)-S(t)\left(u_{0}, v_{0}\right)-\left(D S(t)\left(u_{0}, v_{0}\right)\right)\left(u_{0}^{\prime}, v_{0}^{\prime}\right)\right\|_{H^{2 \alpha}(\Omega) \times H^{2 \beta}(\Omega)}^{2} \\
& \leq C_{29}\left(\left\|u_{0}^{\prime}\right\|_{H^{2 \alpha}(\Omega)}^{2}+\left\|v_{0}^{\prime}\right\|_{H^{2 \beta}(\Omega)}^{2}\right) .
\end{aligned}
$$

Let $\left(U_{1}(t), V_{1}(t)\right),\left(U_{2}(t), V_{2}(t)\right), \cdots,\left(U_{N}(t), V_{N}(t)\right)$ be the solutions of the linear Equation (3.33) corresponding to the initial value $\left(U_{1}(0), V_{1}(0)\right)=\left(\zeta_{1}, \xi_{1}\right), U_{2}(0), V_{2}(0)=\left(\zeta_{2}, \xi_{2}\right), \cdots,\left(U_{N}(0), V_{N}(0)\right)=\left(\zeta_{N}, \xi_{N}\right)$, so there is

$$
\begin{aligned}
& \frac{\mathrm{d}}{\mathrm{d} t}\left\|\left(U_{1}(t), V_{1}(t)\right) \wedge\left(U_{2}(t), V_{2}(t)\right) \wedge \cdots \wedge\left(U_{N}(t), V_{N}(t)\right)\right\|^{2} \\
& +2 \operatorname{tr}\left(L(u(t), v(t)) \cdot Q_{N}\right)\left\|\left(U_{1}(t), V_{1}(t)\right) \wedge\left(U_{2}(t), V_{2}(t)\right) \wedge \cdots \wedge\left(U_{N}(t), V_{N}(t)\right)\right\|^{2}=0 .
\end{aligned}
$$

$L(u(t), v(t))=L\left(S(t)\left(u_{0}, v_{0}\right)\right)$ is linear mapping that is defined in the problem (3.34), $\wedge$ represents the outer product, tr represents the trace, $Q_{N}$ is the orthogonal projection from $L^{2}(\Omega)$ to the span $\left\{\left(U_{1}(t), V_{1}(t)\right),\left(U_{2}(t), V_{2}(t)\right), \cdots,\left(U_{N}(t), V_{N}(t)\right)\right\}$.

Theorem 5. Under the assume of Theorem 3, the global attractor $A$ of problem (1.1) has finite Hausdorff and fractal dimension, and

$$
d_{H} \leq J_{0}, d_{F} \leq 2 J_{0},
$$

Here $J_{0}$ is a minimal positive integer of the following inequality

$$
J_{0}=\frac{1}{2 C^{\prime 2 l}(\gamma+\eta)}\left\{(\gamma+\eta) C^{\prime}+\left[\left((\gamma+\eta) C^{\prime}\right)^{2}+16 C^{\prime 2 l}(\gamma+\eta)+C_{49}\right]^{\frac{1}{2}}\right\},
$$

Proof. By theorem [8], we need to estimate the lower bound of $\operatorname{tr}\left(L(u(t), v(t)) \cdot Q_{N}\right)$. Let $\left(\varphi_{1}, \psi_{1}\right),\left(\varphi_{2}, \psi_{2}\right), \cdots,\left(\varphi_{N}, \psi_{N}\right)$ be the orthogonal basis of subspace of $Q_{N} L^{2}(\Omega)$,

$$
\begin{aligned}
\operatorname{tr}\left(L(u(t), v(t)) \cdot Q_{N}\right)= & \sum_{j=1}^{N}\left\{\left(L(u(t)) \varphi_{j}, \varphi_{j}\right)\right\}+\sum_{j=1}^{N}\left\{\left(L(v(t)) \psi_{j}, \psi_{j}\right)\right\} \\
= & \sum_{j=1}^{N}\left\{\left(\varphi_{j} \nabla u+u \nabla \varphi_{j}-\psi_{j} \nabla v-v \nabla \psi_{j}+\gamma A^{2 \alpha} \varphi_{j}, \varphi_{j}\right)\right\} \\
& +\sum_{j=1}^{N}\left\{\left(\varphi_{j} \nabla v+u \nabla \psi_{j}-\psi_{j} \nabla u-v \nabla \varphi_{j}+\eta A^{2 \beta} \psi_{j}, \psi_{j}\right)\right\} \\
= & \sum_{j=1}^{N}\left\{\left(\varphi_{j} \nabla u-\psi_{j} \nabla v-v \nabla \psi_{j}, \varphi_{j}\right)+\gamma\left\|A^{\alpha} \varphi_{j}\right\|^{2}\right\} \\
& +\sum_{j=1}^{N}\left\{\left(\varphi_{j} \nabla v-\psi_{j} \nabla u-v \nabla \varphi_{j}, \psi_{j}\right)+\eta\left\|A^{\beta} \psi_{j}\right\|^{2}\right\}
\end{aligned}
$$


According to (3.8)-(3.9), we can get

$$
\begin{aligned}
& \left|\left(\varphi_{j} \nabla u, \varphi_{j}\right)\right| \leq\left\|\varphi_{j}\right\|\|\nabla u\|\left\|\varphi_{j}\right\|_{\infty} \leq\left\|\varphi_{j}\right\|\left\|A^{\alpha} u\right\|\left\|A^{\alpha} \varphi_{j}\right\| \leq C_{46}\left\|\varphi_{j}\right\|\left\|A^{\alpha} \varphi_{j}\right\| \leq \frac{\gamma}{4}\left\|A^{\alpha} \varphi_{j}\right\|^{2}+\frac{C_{46}^{2}}{\gamma}\left\|\varphi_{j}\right\|^{2} . \\
& \left|\left(\psi_{j} \nabla v, \varphi_{j}\right)\right| \leq\left\|\psi_{j}\right\|\left\|\nabla \varphi_{j}\right\|\|v\|_{\infty} \leq C_{47}\left\|\psi_{j}\right\|\left\|\nabla \varphi_{j}\right\| \leq \frac{\gamma}{4}\left\|\nabla \varphi_{j}\right\|^{2}+\frac{C_{47}^{2}}{\gamma}\left\|\psi_{j}\right\|^{2} . \\
& \left|\left(v \nabla \psi_{j}, \varphi_{j}\right)\right| \leq\left\|\nabla \psi_{j}\right\|\left\|\varphi_{j}\right\|\|v\|_{\infty} \leq C_{47}\left\|\nabla \psi_{j}\right\|\left\|\varphi_{j}\right\| \leq \frac{\eta}{4}\left\|\nabla \psi_{j}\right\|^{2}+\frac{C_{47}^{2}}{\eta}\left\|\varphi_{j}\right\|^{2} . \\
& \left|\left(\varphi_{j} \nabla v, \psi_{j}\right)\right| \leq\left\|\varphi_{j}\right\|\left\|\nabla \psi_{j}\right\|\|v\|_{\infty} \leq C_{47}\left\|\varphi_{j}\right\|\left\|\nabla \psi_{j}\right\| \leq \frac{\eta}{4}\left\|\nabla \psi_{j}\right\|^{2}+\frac{C_{47}^{2}}{\eta}\left\|\varphi_{j}\right\|^{2} . \\
& \left|\left(\psi_{j} \nabla u, \psi_{j}\right)\right| \leq\left\|\psi_{j}\right\|\|\nabla u\|\left\|\psi_{j}\right\|_{\infty} \leq\left\|\psi_{j}\right\|\left\|A^{\alpha} u\right\|\left\|A^{\beta} \psi_{j}\right\| \leq C_{48}\left\|\psi_{j}\right\|\left\|A^{\beta} \psi_{j}\right\| \leq \frac{\eta}{4}\left\|A^{\beta} \psi_{j}\right\|^{2}+\frac{C_{48}^{2}}{\eta}\left\|\psi_{j}\right\|^{2} . \\
& \left|\left(v \nabla \varphi_{j}, \psi_{j}\right)\right| \leq\left\|\nabla \varphi_{j}\right\|\left\|\psi_{j}\right\|\|v\|_{\infty} \leq C_{47}\left\|\nabla \varphi_{j}\right\|\left\|\psi_{j}\right\| \leq \frac{\gamma}{4}\left\|\nabla \varphi_{j}\right\|^{2}+\frac{C_{47}^{2}}{\gamma}\left\|\psi_{j}\right\|^{2} .
\end{aligned}
$$

Under the bounded condition, select $\left(\varphi_{j}(x, y), \psi_{j}(x, y)\right)=\mathrm{e}^{i k_{1} x+i k_{2} y}$ is the standard eigenfunction of $-\Delta u=\lambda u$, $-\Delta v=\lambda \nu$ and the corresponding eigenvalues are $\lambda_{j}(j=1,2, \cdots)$, and

$$
\begin{gathered}
\left\|\nabla \varphi_{j}\right\|^{2}=\left\|\nabla \psi_{j}\right\|^{2}=\lambda_{j},\left\|\Delta \varphi_{j}\right\|^{2}=\left\|\Delta \psi_{j}\right\|^{2}=\lambda_{j}^{2},\left\|\varphi_{j}\right\|^{2}=\left\|\psi_{j}\right\|^{2}=1, \\
\left\|A^{\alpha} \varphi_{j}\right\|^{2}=\lambda_{j}^{2 \alpha},\left\|A^{\beta} \varphi_{j}\right\|^{2}=\lambda_{j}^{2 \beta}, \lambda_{j} \geq\left[\frac{(j-1)^{\frac{1}{2}}}{2}-1\right]^{2} \sim C \cdot j^{\frac{2}{n}} .
\end{gathered}
$$

Let $C_{49} \geq \frac{C_{46}^{2}+2 C_{47}^{2}}{\gamma}+\frac{2 C_{47}^{2}+C_{48}^{2}}{\eta}$. Therefore, we can get

$$
\begin{aligned}
\operatorname{tr}\left(L(u(t), v(t)) \cdot Q_{N}\right) & \geq \gamma \sum_{j=1}^{N} \lambda_{j}^{2 \alpha}+\eta \sum_{j=1}^{N} \lambda_{j}^{2 \beta}-\frac{\gamma}{4} \sum_{j=1}^{N} \lambda_{j}^{2 \alpha}-\frac{\eta}{4} \sum_{j=1}^{N} \lambda_{j}^{2 \beta}-\left(\frac{\gamma}{2}+\frac{\eta}{2}\right) \sum_{j=1}^{N} \lambda_{j}-N C_{49} \\
& \geq \frac{3 \gamma}{4} \sum_{j=1}^{N} \lambda_{j}^{2 \alpha}+\frac{3 \eta}{4} \sum_{j=1}^{N} \lambda_{j}^{2 \beta}-\left(\frac{\gamma}{2}+\frac{\eta}{2}\right) \sum_{j=1}^{N} \lambda_{j}-N C_{49}
\end{aligned}
$$

Let $l=\min \{\alpha, \beta\}$.

By $\lambda_{j} \geq C^{\prime} j^{\frac{2}{n}}$ and $n=2,3$

$$
\operatorname{tr}\left(L(u(t), v(t)) \cdot Q_{N}\right) \geq\left(\frac{3 \gamma}{4}+\frac{3 \eta}{4}\right) \sum_{j=1}^{N} \lambda_{j}^{2 l}-\left(\frac{\gamma}{2}+\frac{\eta}{2}\right) \sum_{j=1}^{N} \lambda_{j}-N C_{49},
$$

$$
\begin{gathered}
\sum_{j=1}^{N} j^{\frac{4 l}{n}} \geq \sum_{j=1}^{N} j^{2}=\frac{N(N+1)(2 N+1)}{6}>\frac{N^{3}}{3}, \\
\sum_{j=1}^{N} j=\frac{N(N+1)}{2}>\frac{N^{2}}{2},
\end{gathered}
$$

So, we can obtain

$$
N>\frac{1}{2 C^{\prime 2 l}(\gamma+\eta)}\left\{(\gamma+\eta) C^{\prime}+\left[\left((\gamma+\eta) C^{\prime}\right)^{2}+16 C^{\prime 2 l}(\gamma+\eta)+C_{49}\right]^{\frac{1}{2}}\right\}=J_{0},
$$

We have 


$$
\operatorname{tr}\left(L(u(t), v(t)) \cdot Q_{N}\right)>0 .
$$

Therefore

$$
d_{H} \leq J_{0}, d_{F} \leq 2 J_{0} .
$$

\section{Funding}

This work is supported by the National Natural Sciences Foundation of People’s Republic of China under Grant 11161057.

\section{References}

[1] Wu, J. (2003) Generalized MHD Equations. Journal of Differential Equations, 195, 284-312. http://dx.doi.org/10.1016/j.jde.2003.07.007

[2] Tran, C.V., Yu, X. andZhai, Z. (2013) On Global Regularity of 2D Generalized Magnetohydrodynamic Equations. Journal of Differential Equations, 254, 4194-4216. http://dx.doi.org/10.1016/j.jde.2013.02.016

[3] Mattingly, J.C. and Sinai, Ya.G. (1999) An Elementary Proof of the Existence and Uniqueness Theorem for the NavierStokes Equations. Communications in Contemporary Mathematics, 1, 497-516. http://dx.doi.org/10.1142/S0219199799000183

[4] Ladyzhenskaya, O.A. and Seregin, G.A. (1960) Mathematical Problems of Hydrodynamics and Magnetohydrodynamics of a Viscous Incompressible Fluid. Proceedings of V.A. Steklov Mathematical Institute, 59, 115-173. (In Russian)

[5] Caffarelli, L., Kohn, R.V. and Nirenberg, L. (1982) Partial Regularity of Suitable Weak Solutions of the Navier-Stokes Equations. Communications on Pure and Applied Mathematics, 35, 771-831. http://dx.doi.org/10.1002/cpa.3160350604

[6] Vialov, V. (2014) On the Regularity of Weak Solutions to the MHD System near the Boundary. Journal of Mathematical Fluid Mechanics, 16, 745-769. http://dx.doi.org/10.1007/s00021-014-0184-3

[7] Ladyzhenskaya, O.A. and Seregin, G.A. (1999) On Partial Regularity of Suitable Weak Solutions to the ThreeDimensional Navier-Stokes Equations. Journal of Mathematical Fluid Mechanics, 1, 356-387. http://dx.doi.org/10.1007/s000210050015

[8] Scheffer, V. (1977) Hausdorff Measure and Navier-Stokes Equations. Communications in Mathematical Physics, 55, 97-112. http://dx.doi.org/10.1007/BF01626512

[9] Lin, G.G. (2011) Nonlinear Evolution Equations. Yunnan University Press, Kunming. 